\title{
Speed Control of a Doubly Fed Induction Motor using Fuzzy Logic Techniques
}

\author{
D. Ben Attous and Y. Bekakra \\ Department of Electrical Engineering, El-Oued University Center. \\ dbenattous@yahoo.com, youcef1984@gmail.com
}

\begin{abstract}
This paper presents a comparison between a fuzzy logic controller and a conventional IP controller used for speed control with a direct stator flux orientation control of a doubly fed induction motor. The effectiveness of the proposed control strategy is evaluated under different operating conditions such as of reference speed and for load torque step changes at nominal parameters and in the presence of parameter variation. Simulation results show that the fuzzy logic controller is more robust than a conventional IP controller against parameter variation and uncertainty, and is less sensitive to external load torque disturbance with a fast dynamic response.
\end{abstract}

Keywords: direct stator flux orientation control, doubly fed induction motor, fuzzy logic controller, Fuzzy PI controller, conventional IP controller.

\section{Introduction}

Due to many freedom degrees, the Doubly Fed Induction Machine (DFIM) presents several advantages as well as motor application in high power applications such as traction, marine propulsion or as generator in wind energy conversion systems like wind turbine, or pumped storage systems [1].

The DFIM has some distinct advantages compared to the conventional squirrel-cage machine. The DFIM can be fed and controlled stator or rotor by various possible combinations. Indeed, the input-commands are done by means of four precise degrees of control freedom relatively to the squirrel cage induction machine where its control appears quite simpler. The flux orientation strategy can transform the non linear and coupled DFIM-mathematical model to a linear model conducting to one attractive solution as well as under generating or motoring operations [2], [3].

Several methods of control are used to control the induction motor among which the vector control or field orientation control that allows a decoupling between the torque and the flux, in order to obtain an independent control of torque and the flux like DC motors [4].

Therefore, decoupling the control scheme is required by compensation of the coupling effect between q-axis and d-axis current dynamics [4].

With the field orientation control (FOC) method, induction machine drives are becoming a major candidate in high-performance motion control applications, where servo quality operation is required. Fast transient response is made possible by decoupled torque and flux control. The most widely used control method is perhaps the proportional integral control (PI). It is easy to design and implement, but it has difficulty in dealing with parameter variations, and load disturbances [5]. Recent literature has paid much attention to the potential of fuzzy control in machine drive applications.

In the area of the control of the electric machines, the research works are oriented more and more towards the application of the modern control techniques. These techniques involve in a vertiginous way with the evolution of the computers and power electronics. This allows to lead to the industrial processes of high performances. These techniques are the fuzzy control, the adaptive control, the sliding mode control etc [6].

Received: February 8, 2010. Accepted: July 8,2010 
Fuzzy theory was first proposed and investigated by Prof. Zadeh in 1965. The Mamdani fuzzy inference system was presented to control a steam engine and boiler combination by linguistic rules. Fuzzy logic is expressed by means of if-then rules with the human language.

In the design of a fuzzy logic controller, the mathematical model is not necessary. Therefore, the fuzzy logic controller is of good robustness. Owing to its easy application, it has been widely used in industry. However, the rules and the membership functions of a fuzzy logic controller are based on expert experience or knowledge database [7].

In this study, we suggest a control scheme to achieve the goal of speed regulation with direct stator flux orientation control DFIM drive and fuzzy logic control (Fuzzy-PI).

\section{Machine STATE-EQUATIONS}

The electrical model of the DFIM is expressed in a (d-q) synchronous rotating frame.

The control is done under the following considerations [8]:

An input-output current decoupling is set for all currents;

The (d-q) frame is oriented with the stator flux;

Due to the large gap between the mechanical and electrical time constants, the speed can be considered as invariant with respect to the state vector.

Under these conditions, the electrical equations of the machine are described by a time variant state space system as shown in (1):

$$
\begin{aligned}
\dot{X} & =A . X+B . U \\
Y & =C \cdot X
\end{aligned}
$$

With,

$$
\begin{aligned}
& X=\left[\begin{array}{llll}
i_{s d} & i_{s q} & i_{r d} & i_{r q}
\end{array}\right]^{T} \\
& U=\left[V_{s d} V_{s q} V_{r d} V_{r q}\right]^{T} \\
& A=\left[\begin{array}{cccc}
-a_{1} & a \omega+\omega_{s} & a_{3} & a_{5} \omega \\
-a \omega-\omega_{s} & -a_{1} & -a_{5} \omega & a_{3} \\
a_{4} & -a_{6} \omega & -a_{2} & -\frac{\omega}{\sigma}+\omega_{s} \\
a_{6} \omega & a_{4} & \frac{\omega}{\sigma}-\omega_{s} & -a_{2}
\end{array}\right], \quad B=\left[\begin{array}{cccc}
b_{1} & 0 & -b_{3} & 0 \\
0 & b_{1} & 0 & -b_{3} \\
-b_{3} & 0 & b_{2} & 0 \\
0 & -b_{3} & 0 & b_{2}
\end{array}\right], \\
& C=\left[\begin{array}{llll}
1 & 0 & 0 & 0 \\
0 & 1 & 0 & 0 \\
0 & 0 & 1 & 0 \\
0 & 0 & 0 & 1
\end{array}\right]
\end{aligned}
$$

Where:

$$
\begin{aligned}
& a=\frac{1-\sigma}{\sigma}, \quad a_{1}=\frac{R_{s}}{\sigma L_{s}}, \quad a_{2}=\frac{R_{r}}{\sigma L_{r}}, \quad a_{3}=\frac{R_{r} M}{\sigma L_{s} L_{r}}, \quad a_{4}=\frac{R_{s} M}{\sigma L_{s} L_{r}}, \quad a_{5}=\frac{M}{\sigma L_{s}}, \\
& a_{6}=\frac{M}{\sigma L_{r}}, b_{1}=\frac{1}{\sigma L_{s}}, b_{2}=\frac{1}{\sigma L_{r}}, b_{3}=\frac{M}{\sigma L_{s} L_{r}}, \sigma=1-\frac{M^{2}}{L_{s} L_{r}}
\end{aligned}
$$


The mechanical equation is expressed by (2):

$$
J \frac{d \Omega}{d t}=C_{e}-C_{r}-f \Omega
$$

The electromagnetic torque is given by [8]:

$$
C_{e}=\frac{P M}{L_{s}}\left(\phi_{s q} \cdot i_{r d}-\phi_{s d} . i_{r q}\right)
$$

\section{Direct Stator Flux Orientation Control}

In this section, the DFIM model can be described by the following state equations in the synchronous reference frame whose axis $d$ is aligned with the stator flux vector [9], [10]:

$$
\begin{aligned}
& \phi_{s d}=\phi_{s} ; \frac{d \phi_{s q}}{d t}=\phi_{s q}=0 \\
& i_{r d}=\frac{\phi_{s}^{*}}{M} \\
& i_{r q}=-\frac{L_{s}}{P M \phi_{s}^{*}} C_{e}^{*} \\
& \frac{d \theta_{s}}{d t}=\omega_{s}=\left(\frac{R_{s} \cdot M}{L_{s}} i_{r q}+V_{s q}\right) / \phi_{s}^{*} \\
& V_{r d}=\left(R_{r}+\frac{M^{2}}{L_{s} T_{s}}\right) i_{r d}+\sigma L_{r} \frac{d i_{r d}}{d t}+\frac{M}{L_{s}} V_{s d}-\frac{M}{L_{s} T_{s}} \phi_{s d}-\sigma L_{r}\left(\omega_{s}-\omega\right) i_{r q}
\end{aligned}
$$

Substituting the equation (5) in equation (8), we obtain:

$$
\begin{aligned}
& V_{r d}=R_{r} i_{r d}+\sigma L_{r} \frac{d i_{r d}}{d t}+\frac{M}{L_{s}} V_{s d}-\sigma L_{r}\left(\omega_{s}-\omega\right) i_{r q} \\
& V_{r q}=\left(R_{r}+\frac{M^{2}}{L_{s} T_{s}}\right) i_{r q}+\sigma L_{r} \frac{d i_{r q}}{d t}+\frac{M}{L_{s}} V_{s q}-\frac{M}{L_{s}} \omega \phi_{s d}+\sigma L_{r}\left(\omega_{s}-\omega\right) i_{r d}
\end{aligned}
$$

With:

$$
T_{r}=\frac{L_{r}}{R_{r}}, T_{s}=\frac{L_{s}}{R_{s}} .
$$

\section{Stator Flux Estimator}

For the DSFOC of DFIM, accurate knowledge of the magnitude and position of the stator flux vector is necessary. In a DFIM motor mode, as stator and rotor current are measurable, the stator flux can be estimated (calculate). The flux estimator can be obtained by the following equations [10]:

$$
\begin{aligned}
& \phi_{s d}=L_{s} i_{s d}+M i_{r d} \\
& \phi_{s q}=L_{s} i_{s q}+M i_{r q}
\end{aligned}
$$


D. Ben Attous, et al.

The position stator flux is calculated by the following equations:

$$
\theta_{r}=\theta_{s}-\theta
$$

In which:

$$
\theta_{s}=\int \omega_{s} d t, \theta=\int \omega d t, \omega=P \Omega .
$$

\section{Fuzzy Logic Controller}

The structure of a complete fuzzy control system is composed from the following blocs: Fuzzification, Knowledge base, Inference engine, Defuzzification. Figure 1 shows the structure of a fuzzy logic controller.

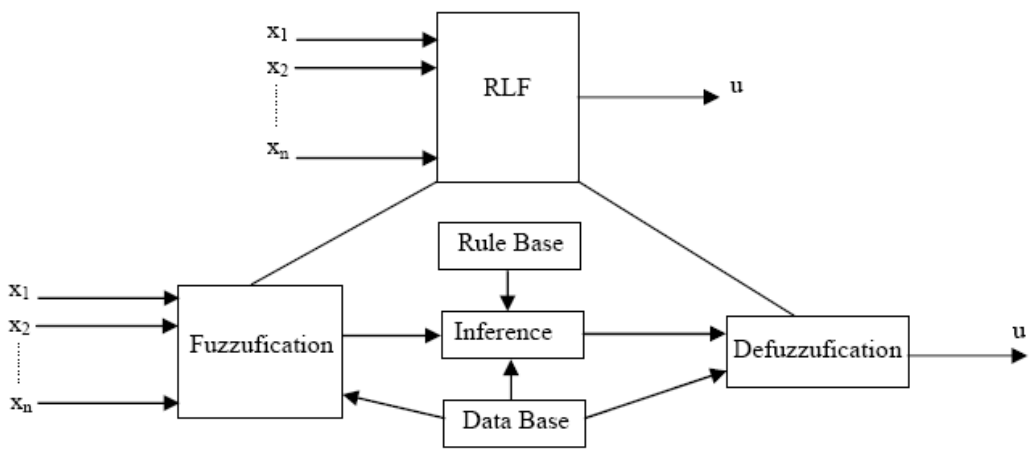

Figure 1. The structure of a fuzzy logic controller

The fuzzification module converts the crisp values of the control inputs into fuzzy values. A fuzzy variable has values, which are defined by linguistic variables (fuzzy sets or subsets) such as low, medium, high, slow... where each is defined by gradually varying membership function. In fuzzy set terminology, all the possible values that a variable can assume are named universe of discourse, and fuzzy sets (characterized by membership function) cover whole universe of discourse. The shape fuzzy sets can be triangular, trapezoidal, etc [11].

A fuzzy control essentially embeds the intuition and experience of a human operator, and sometimes those of a designer and researcher. The data base and the rules form the knowledge base which is used to obtain the inference relation $\mathrm{R}$. The data base contains a description of input and output variables using fuzzy sets. The rule base is essentially the control strategy of the system. It is usually obtained from expert knowledge or heuristics; it contains a collection of fuzzy conditional statements expressed as a set of IF-THEN rules, such as:

$$
\begin{gathered}
\mathrm{R}^{(\mathrm{i})} \text { : If } \mathrm{x}_{1} \text { is } \mathrm{F} 1 \text { and } \mathrm{x}_{2} \text { is } \mathrm{F} 2 \ldots \text { and } \mathrm{x}_{\mathrm{n}} \text { is } \mathrm{Fn} \\
\text { THEN } \mathrm{Y} \text { is } \mathrm{G}^{(\mathrm{i})}, \mathrm{i}=1, \ldots, \mathrm{M}
\end{gathered}
$$

Where: $\left(x_{1}, x_{2}, \ldots, x_{n}\right)$ is the input variables vector, $Y$ is the control variable, $M$ is the number of rules, $n$ is the number fuzzy variables, $\left(F_{1}, F_{2}, \ldots F_{n}\right)$ are the fuzzy sets.

For the given rule base of a control system, the fuzzy controller determines the rule base to be fired for the specific input signal condition and then computes the effective control action (the output fuzzy variable) [11].

The composition operation is the method by which such a control output can be generated using the rule base. Several composition methods, such as max-min or sup-min and max-dot have been proposed in the literature. 
The mathematical procedure of converting fuzzy values into crisp values is known as 'defuzzification'. A number of defuzzification methods have been suggested. The choice of defuzzification methods usually depends on the application and the available processing power. This operation can be performed by several methods of which center of gravity (or centroid) and height methods are common [11].

\section{A. Fuzzy-PI Controller}

The fuzzy controller is basically an input/ output static non-linear mapping, the controller action can be written in the form [13]:

$$
u=k_{e} . e+k_{c e} . c e
$$

The Fuzzy-PI output is:

$$
y=k_{p} \cdot u+\int k_{i} \cdot u
$$

Where: $k_{e}$ is the gain of the speed error, $k_{c e}$ is the gain of the change of speed error, $k_{p}$ is the proportional factor; $k_{i}$ is the integral factor, $e$ is the speed error, $c e$ is the change of speed error, $u$ is the fuzzy output.

The Fuzzy-PI controller in a vector-control of DFIM is used as presented in Figure 2.

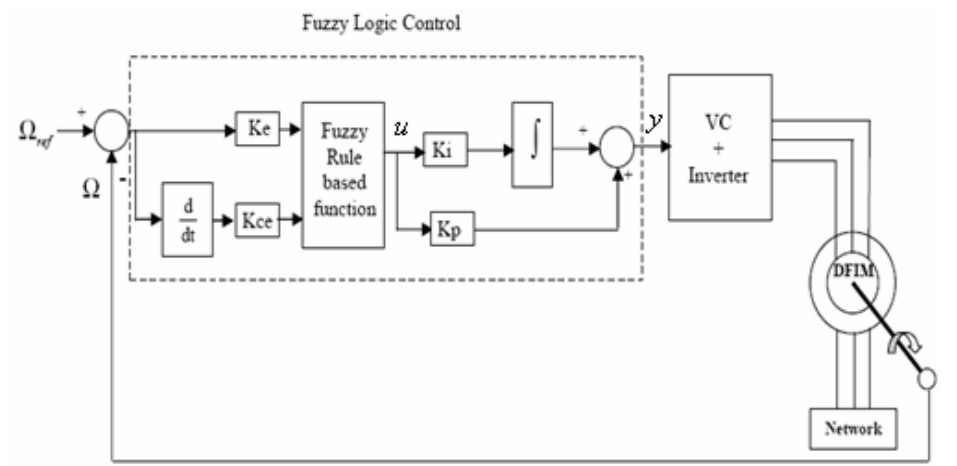

Figure 2. Block diagram of vector-control of DFIM using Fuzzy-PI controller

\section{B. Knowledge Base Proposed}

Figure 3 and 4 shows respectively the triangle-shaped membership functions of error $(e)$ and change of error ( $c e$ ). The fuzzy sets are designated by the labels: NB (negative big), NM (negative medium), NS (negative small), Z (zero), PS (positive small), PM (positive medium), PB (positive big), NVS (negative very small) and PVS (positive very small).

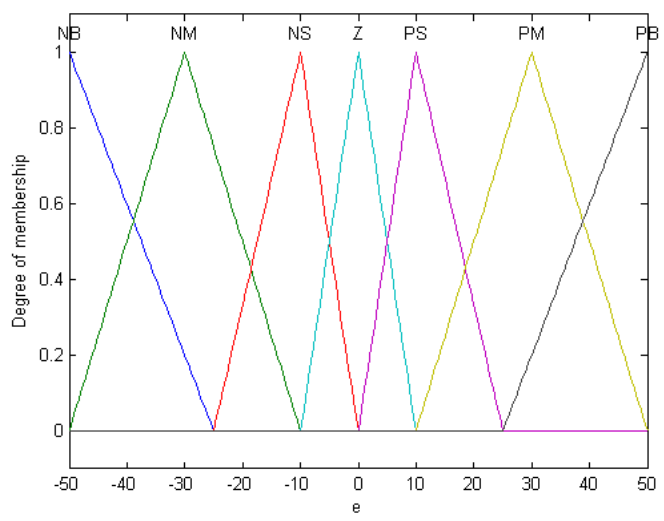

Figure 3. Membership functions for input e 
D. Ben Attous, et al.

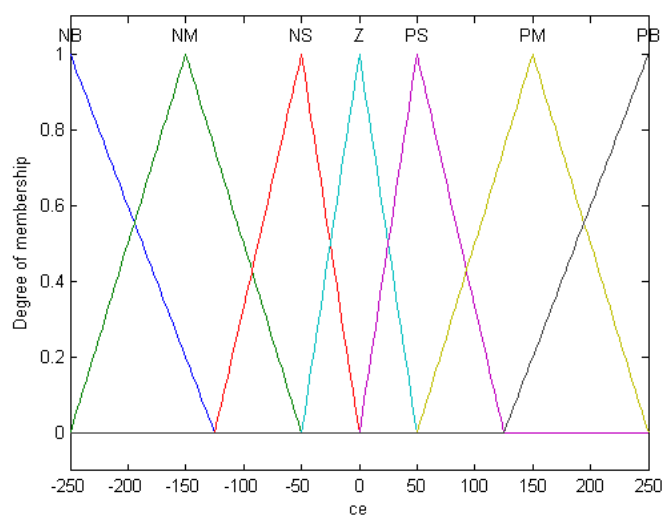

Figure 4. Membership functions for input ce

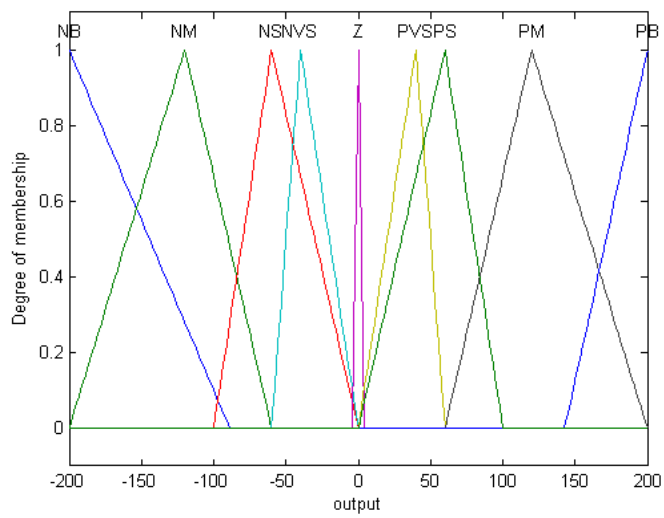

Figure 5. Membership functions for output

Figure 5 shows the proposed membership functions for output variable.

In this paper, the triangular membership function, the max-min reasoning method, and the center of gravity defuzzification method are used, as those methods are most frequently used in many literatures [11]. The inference strategy used in this system is the Mamdani algorithm.

Table 1. Linguistic Rule Table

\begin{tabular}{|l|l|l|l|l|l|l|l|}
\hline $\begin{array}{l}\text { ce } \\
\text { ce }\end{array}$ & NB & NM & NS & Z & PS & PM & PB \\
\hline NB & NB & NB & NB & NM & NS & NVS & Z \\
\hline NM & NB & NB & NM & NS & NVS & Z & PVS \\
\hline NS & NB & NM & NS & NVS & Z & PVS & PS \\
\hline Z & NM & NS & NVS & Z & PVS & PS & PM \\
\hline PS & NS & NVS & Z & PVS & PS & PM & PB \\
\hline PM & NVS & Z & PVS & PS & PM & PB & PB \\
\hline PB & Z & PVS & PS & PM & PB & PB & PB \\
\hline
\end{tabular}


All the membership functions (MFs) are asymmetrical because near the origin (steady state), the signals require more precision. Seven MFs are chosen for e and ce signals and nine for output. All the MFs are symmetrical for positive and negative values of the variables. Thus, maximum $7 \times 7=49$ rules can be formed as tabulated in Table I, [12].

\section{Results and Discussion}

The DFIM used in this work is a $0.8 \mathrm{~kW}$, whose nominal parameters are reported in appendix.

The Fuzzy-PI controller in a DSFOC drive system as presented in Figure 2.

The Figure 6 presents the block diagram of fuzzy logic control of the DFIM using MATLAB/SIMULINK. As shown in this figure, the speed loop uses a fuzzy logic controller (Fuzzy-PI) and the stator flux is controlled by PI controller. The block "command system by PI controller" is the vector control, which to produce the quadrature-axis rotor current reference which controls the motor torque. The motor flux is controlled by direct-axis rotor current reference. Block "Park inversion" is used to convert voltage reference $V_{r d}$ and $V_{r q}$ into voltage reference $V_{a}, V_{b}$ and $V_{c}$ for introduce in "PMW inverter". Motor torque, rotor current, flux and speed signals are available at the output of the "Model of DFIM".

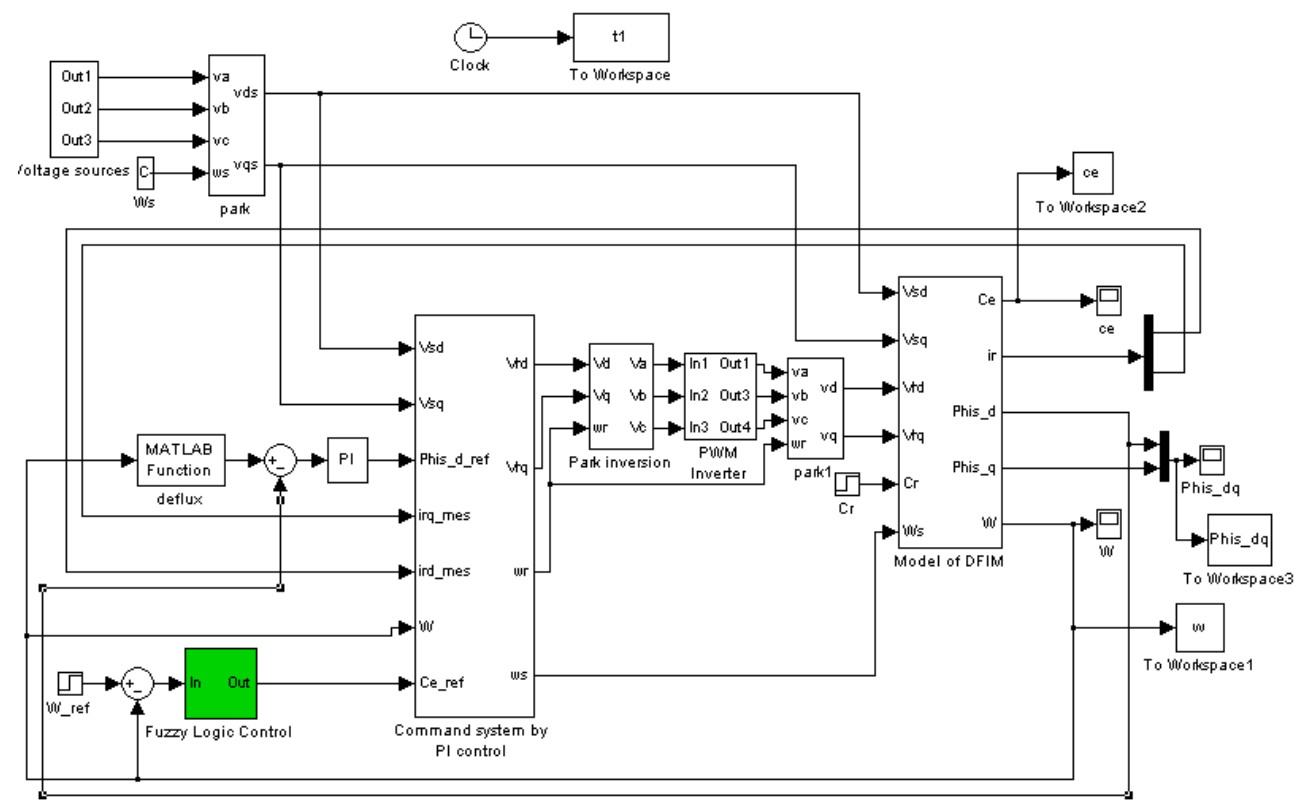

Figure 6. Block diagram of fuzzy logic control of the DFIM using MATLAB/SIMULINK

\section{A. Load Variation}

In the first test, a cyclic change of different load torque levels are subjected to the motor at certain times and as followings:

Time $=\left[\begin{array}{lllllllllll}0 & 0.8 & 0.8 & 1.1 & 1.1 & 1.4 & 1.4 & 1.7 & 1.7\end{array}\right]$

Torque $=\left[\begin{array}{lllllllll}0 & 0 & 5 & 5 & 3 & 3 & -3 & -3 & 0\end{array}\right] ;$

$\mathrm{T}$ he responses of speed, torque, stator flux and rotor current are shown in Figure 7. The Fuzzy-PI regulator shows the good performances to achieve tracking of the desired trajectory.

At these changes of loads, the Fuzzy-PI regulator rejects the load disturbance very rapidly with no overshoot and with a negligible static error as can be seen in the response of speed (see Figure 7). The decoupling of torque-flux is maintained in permanent mode.

We can see the control is robust from the point of view load variation. 
In order to compare the performance of Fuzzy-PI regulator with another regulator in the same test, the Figure 8 shows the simulated results comparison of traditional IP (Integral Proportional) and Fuzzy-PI regulators of speed control of DFIM under load variation. The Fuzzy PI controller based drive system can handle the sudden change in load torque without overshoot and undershoot and steady state error, whereas the IP controller has steady state error and the response is not as fast as compared to Fuzzy PI controller. Thus the proposed controller has been found superior to the conventional IP controller.

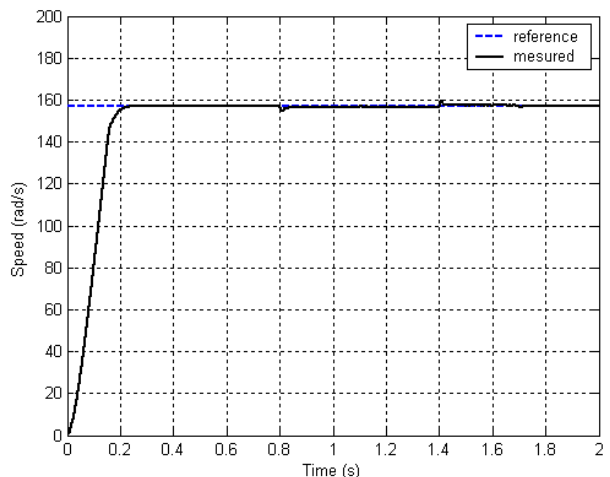

(a)

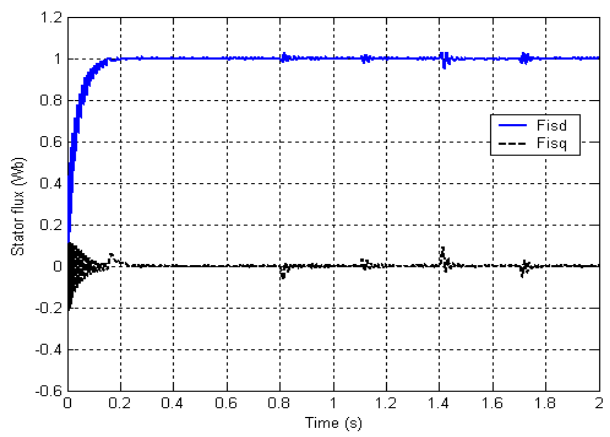

(c)

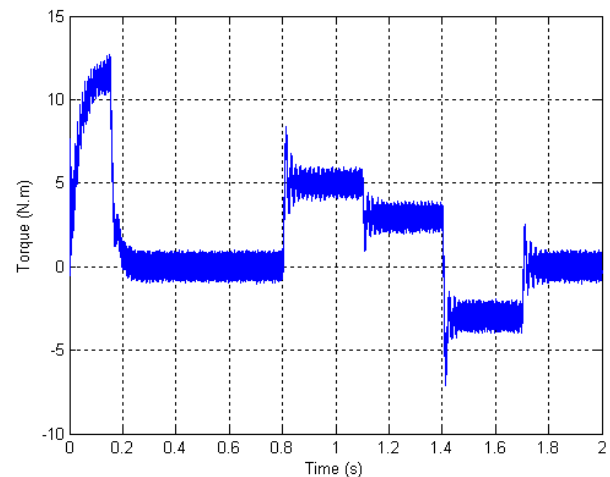

(b)

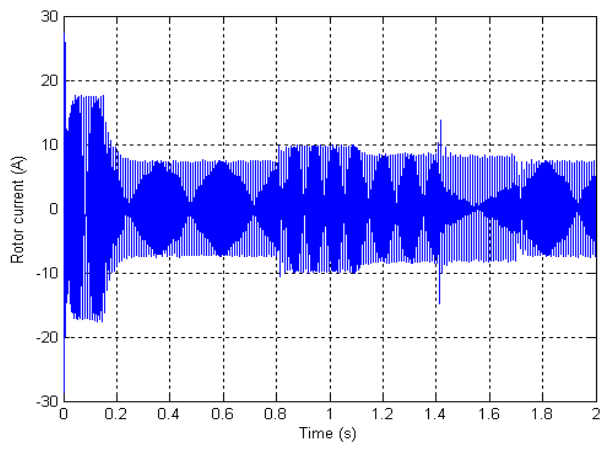

(d)

Figure 7. Results of speed (a), torque (b), stator flux (c), and rotor (d)Rotor current under load variation

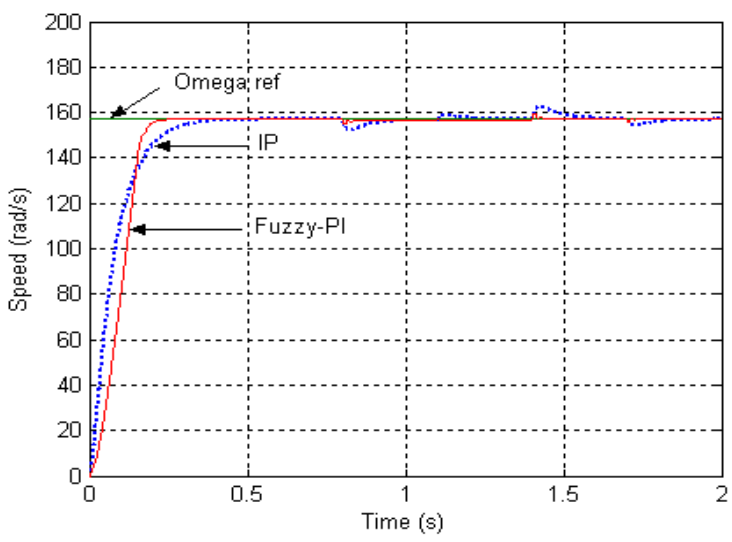

Figure 8. Simulated results comparison of IP and Fuzzy-PI regulators of speed control of DFIM under load variation. 


\section{B. Speed Reversal of Rated Value}

In the second test, speed reversal of $(157,-157 \mathrm{rad} / \mathrm{s})$, with a load of $5 \mathrm{~N} . \mathrm{m}$ applied at $\mathrm{t}=0.6 \mathrm{~s}$.

Figure 9 presents the responses of speed, torque, stator flux and rotor current versus time under speed reference reversal. The speed follows its reference value without overshooting. Figure 10 shows the result under the steady state and the transient condition i.e. step change in load torque at $\mathrm{t}=0.6 \mathrm{~s}$ and speed reversal stage at $\mathrm{t}=1 \mathrm{~s}$ for the Fuzzy PI controller and conventional IP controller from which it can be see that the speed reached the rated value in very short period for the Fuzzy PI controller. .

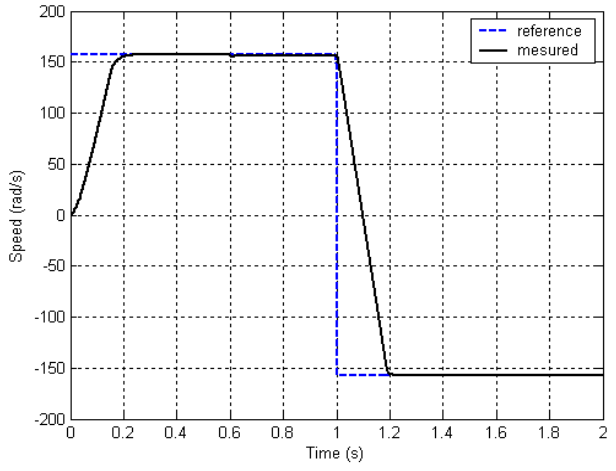

(a)

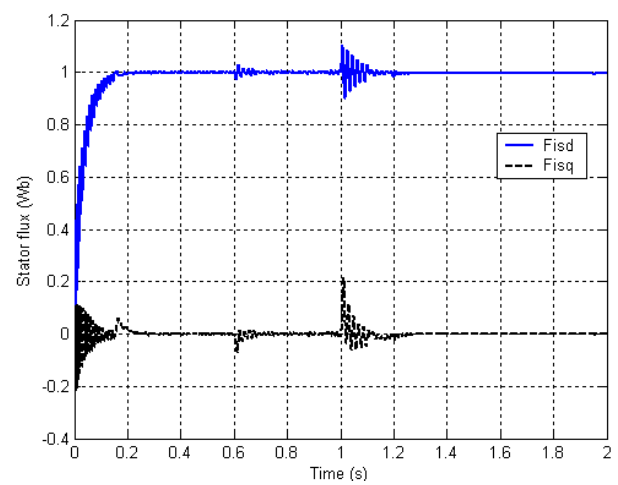

(c)

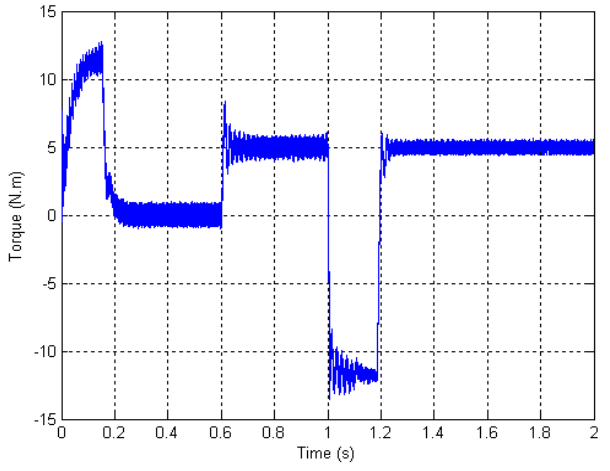

(b)

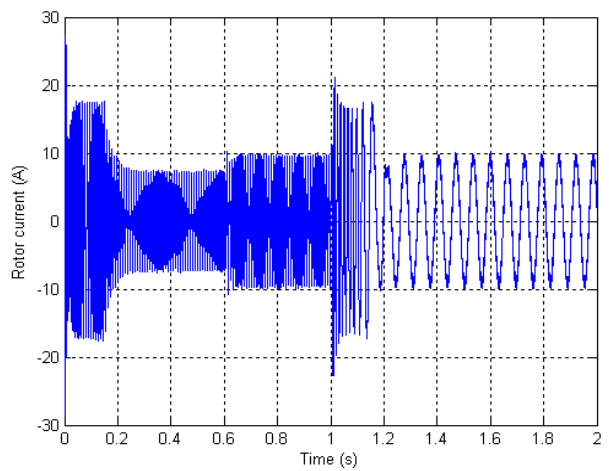

(d)

Figure 9. Results of speed (a), torque (b), stator flux (c), and rotor current (d) under reversal speed

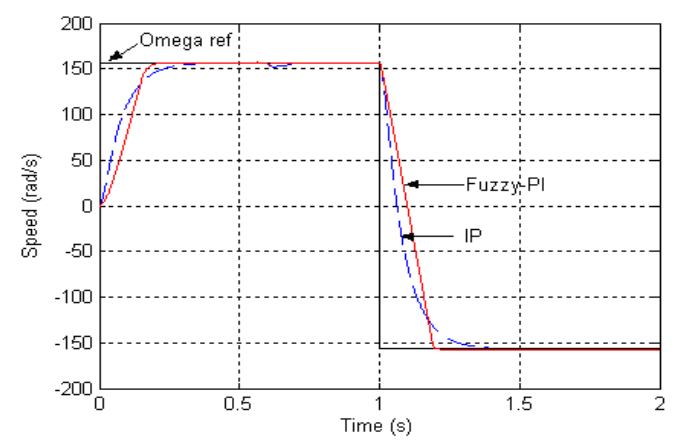

Figure 10. Simulated results comparison of IP and Fuzzy-PI regulators of speed control of DFIM under reversal speed. 
D. Ben Attous, et al.

\section{Robust Control for Different Values of Rotor Resistance}

In order to verifier the robustness of Fuzzy-PI regulator under motor parameters variations, we have simulated the system with different values of the parameter considered and compared to nominal value (real value), one case is considered:

The rotor resistance variations (increase at $50 \%$ of nominal value rotor resistance).

Figure 11 shows the responses speed, torque and stator flux in the test of robustness for different values of rotor resistance.

The results indicate that the Fuzzy-PI regulator is insensitive to the rotor resistance change, which results in the no influence on the torque and stator flux.

For the robustness of control, an increase of the resistance does not have any effect on the performances of the proposed controller.

The fuzzy control gives to our controller a great place towards the control of the system with unknown parameters.

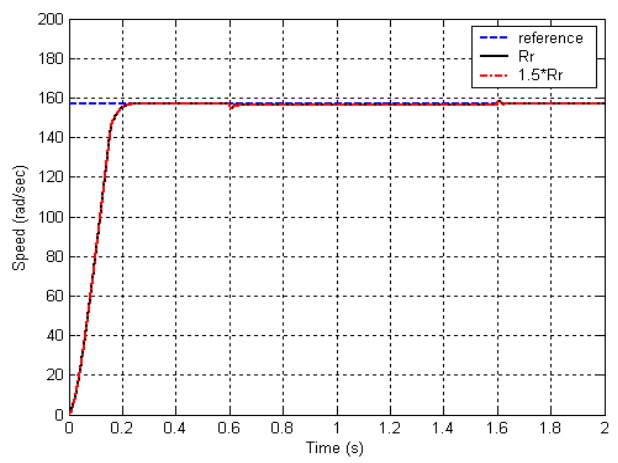

(a)

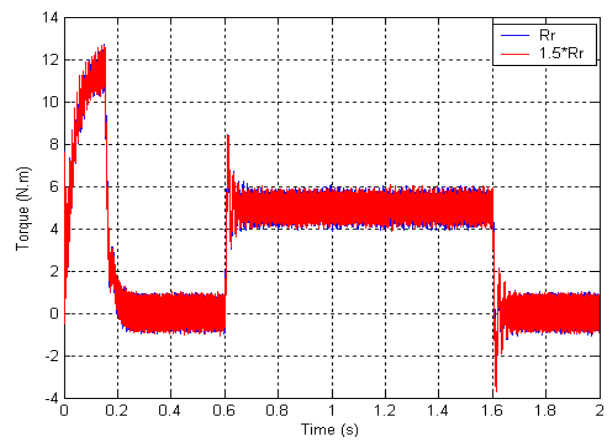

(b)

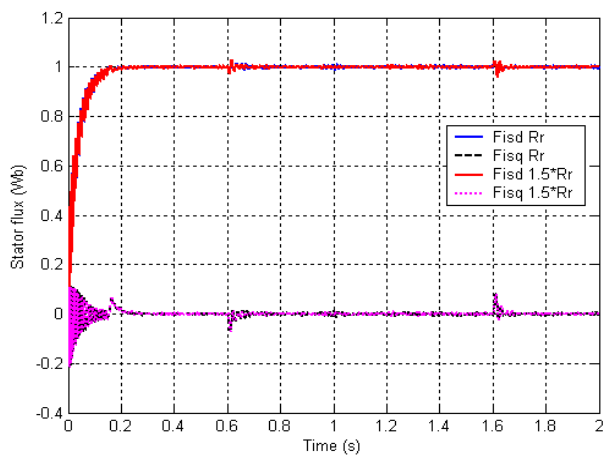

(c)

Figure 11. Test of robustness result for rotor speed (a), torque (b), and stator fue(c) for different values of rotor resistance: nominal case and $+50 \%$ 


\section{Conclusion}

In this paper, we have proposed a fuzzy logic controller for the speed control of doubly fed induction motor with a direct stator flux orientation control. The effectiveness of the proposed controller has been tested on DFIM in comparison with conventional IP controller under different operating conditions. The fuzzy PI regulator proves robustness against rotor resistance variation and insensitivity to load torque disturbance as well as faster dynamics with negligible steady state error at all dynamic operating conditions. Simulation results have shown correct stator flux oriented control behaviour and speed tracking performances.

\section{Appendix}

$i_{r d}, i_{r q}$ : Rotor current components,

$\phi_{s d}, \phi_{s q}$ : Stator flux components,

$V_{s d}, V_{s q}$ : Stator voltage components,

$V_{r d}, V_{r q}$ : Rotor voltage components.

$R_{s}, R_{r}:$ Stator and rotor resistances,

$L_{s}, L_{s}:$ Stator and rotor inductances,

$M$ : Mutual inductance,

$\sigma$ : Leakage factor,

$P$ : Number of pole pairs,

$C_{e}$ : The electromagnetic torque,

$C_{r}$ : The load torque,

$J$ : The moment of inertia,

$\Omega$ : Mechanical speed,

$\omega_{s}, \omega:$ The stator pulsation,

$f$ : The friction coefficient,

$T_{s}, T_{r}$ : Statoric and rotoric time-constant.

$\theta_{s}:$ The electrical stator position,

$\theta$ : The electrical rotor position.

Rated Data of the simulated doubly fed induction motor:

Rated values: 0.8 KW; 220/380 V-50 Hz; 3.8/2.2 A, 1420 rpm.

Rated parameters:

$R_{S}=11.98 \Omega$

$R_{r}=0.904 \Omega$

$L_{\mathrm{s}}=0.414 \mathrm{H}$

$L_{r}=0.0556 \mathrm{H}$

$M=0.126 \mathrm{H}$

$P=2.0$

Mechanical constants:

$J=0.01 \mathrm{Kg} . \mathrm{m}^{2}$

$f=0.00$ I.S. 
D. Ben Attous, et al.

\section{References}

[1] S. Khojet, E. I. Slama-Belkhodja, M. Pietrzak-David, B. de Fornel, "A Fault Tolerant Operating System in a Doubly Fed Induction Machine under Inverter Short-circuit Faults," 1-4244-0136-4/06/\$20.00’ 2006 IEEE, pp 1125-1130.

[2] S. Drid, M. Tadjine and M. S. Naît-Saîd, "Robust backstepping vector control for the doubly fed induction motor," The Institution of Engineering and Technology, Control Theory Appl., 2007, 1, (4), pp. 861-868.

[3] S. Drid, M. Tadjine, M.S. Nait-Said, "Nonlinear feedback control and torque optimization of a doubly fed induction motor," Journal of Electrical Engineering, Vol. 56, NO. 3-4, 2005, 57-63.

[4] Chaari, M. Soltani, M. Gossa, "Comparative study between the conventional regulators and fuzzy logic controller: application on the induction machine," International Journal of Sciences and Techniques of Automatic control \& computer engineering IJ-STA, Volume 1, N², December 2007, pp. 196-212.

[5] Hebert, L. Xu and Y. Tang, "Fuzzy Logic Enhanced Speed Control of an Indirect FieldOriented Induction Machine Drive," IEEE Trans. Power Electronics. Vol. 12. NO 5. September 1997, pp 772-778.

[6] Y. Harbouche, L. Khettache and R. Abdessemed, "Sliding Mode Control of the Double Fed Asynchronous Machine Applayed by Current Sources," Asian Journal of Information Technology 6 (3), pp 362-368, 2007.

[7] B. Allaoua, A. Abderrahmani B. Gasbaoui, A. Nasri, "The Efficiency of Particle Swarm Optimization Applied on Fuzzy Logic DC Motor Speed Control," Serbian Journal of Electrical Engineering Vol. 5, No. 2, November 2008, 247-262.

[8] G. SAlloum, R. MBAYED, M. DE FORNEL B. PIETRZAK-DAVID, "Mixed Sensitivity Ho Control of Doubly Fed Induction Motor," pp 1300-1304, 1-4244-07559/07/\$20.00 C2007 IEEE, pp 1300-1304.

[9] J. Soltani, A. Farrokh Payam, M.A. Abbasian, "A Speed Sensorless Sliding-Mode Controller for Doubly-Fed Induction Machine Drives with Adaptive Backstepping Observer," 1-4244-0726-5/06/\$20.0O '2006 IEEE, pp 2725-2730.

[10] Y. Bekakra, D. Ben attous, "A Sliding Mode Speed and Flux Control of a Doubly Fed Induction Machine," Electrical and Electronics Engineering, 2009. ELECO'2009. International Conference on, 5-8 Nov. 2009, Bursa, pp I-174 - I-178.

[11] Aissaoui, M. Abid, H. Abid, A. Tahour, A. Zeblah, "A Fuzzy Logic Controller for Synchronous Machine,” Journal of Electrical Engineering, Vol. 58, NO. 5, 2007, pp 285290.

[12] V. Kumar, R.R.Joshi, "Hybrid Controller based Intelligent Speed Control of Induction Motor," Journal of Theoretical and Applied Information Technology, pp 71-75, 2005. 


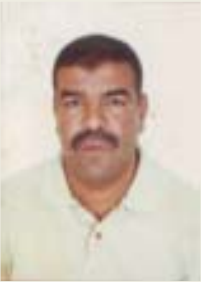

D. Ben Attous was born on May 24, 1959 El Oued Algeria. He received his Engineerning degree in Electrotechnics from Polytechnic National Institute Algiers Algeria in 1984. He got Msc degree in Power Systems from UMIST England in 1987. In 2000, he received his "doctorat d'état" (PhD from Batna university Algeria). He is currently associate professor at El Oued university center Algeria in Electrical Engineering. His research interests in Planning and Economic of Electric Energy System, Optimization Theory and its applications and he also investigated questions related with Electrical Drives and Process Control. He is member of the VTRS research Laboratory.

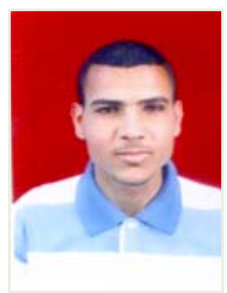

Y. Bekakra was born on Apr28, 1984 in El Oued Algeria. He received his Engineerning degree in Electrotechnics from El Oued university center Algeria in 2007. He prepared his Msc in Power System. His area of research interest is mainly Electrical Drives and Process Control. 